\title{
The Ciliary Marginal Zone of the Retina of Austrolebias charrua Fish is a Highly Proliferative Neurogenic Niche.
}

\author{
Anabel S Fernández, ${ }^{1,3}$ Juan Carlos Rosillo ${ }^{1,2}$, Inés Berrosteguieta ${ }^{1}$, María Laura Herrera ${ }^{1}$ Maximiliano \\ Torres-Pérez ${ }^{1}$, \\ ${ }^{1}$ Depto. Neurociencias Integrativas y Computacionales-Neuroanatomía Comparada, Instituto de Investigaciones Biológicas \\ Clemente Estable (IIBCE), Montevideo (MVD), Uruguay (UY). \\ ${ }^{2}$ Depto. Histología y Embriología, Facultad de Medicina MVD, (UY). \\ ${ }^{3}$ Neuroanatomía Comparada, Unidad Asociada a la Facultad de Ciencias, UdelaR, MVD, UY. Email: * $\underline{\text { afernandez }}$ \\ @iibce.edu.uy, anabelsonia@gmail.com
}

The retinal proliferative activity in fish is a persistent mechanism of plasticity during growth (1). Our previous studies demonstrated that Austrolebias charrua annual fish is an excellent model to study adult brain cell proliferation and neurogenesis (2). These fish present an active and fast neurogenesis in several regions during their short lifespan. The generation of the new neurons in the retina occurs from the annulus in the margin called the Ciliary Marginal Zone (CMZ), considered a neurogenic niche that is able to maintain and regenerate all the cells of the retina after an injury in adulthood (3). To study the proliferation of CMZ in the annual fish, we used three thymidine halogenated analogs to detect cell proliferation at different survival times: 5-chloro-2 -deoxyuridine (CldU) at 1 day and 5-iodo-2-deoxyuridine (IdU) at 30 days (2). We repeated the same experiment using only bromo-2 deoxyuridine at 30 days. We were able to analyze the cell proliferation rate in the ciliary marginal zone, as well as determining populations of cellular progenitors and putative stem cells, through the use of stem cell markers such as Sox 2, Pax6 and BlBP. The neurogenic activity was detected by HuC / D and Beta Tubulin. The analysis by means of transmission electron microscopy allowed the correlation of the existence of at least four different cell populations of progenitors grouped in three sectors of the ciliary marginal zone and another located in the non-pigmentary epithelium.

The quantification of the labeled nuclei with both proliferation markers, indicates that the proportion of putative stem cells that capture two proliferation markers (yellows) without migration separated for a temporal window of 30 days is approximately $20 \%$, two times the rate of the brain.

[1] D.C. Otteson, P. F Hitchcock, Vision research, 43(8) (2003) p. 927.

[2] A. S Fernández et al., Neuroscience. 189 (2011) p. 12.

[3] Y. Wanet et al., Development. 143 (7) (2016), p. 1099.

[4] The authors acknowledge to PEDECIBA and ANII for the contribution and partial financial support for developing this work. 


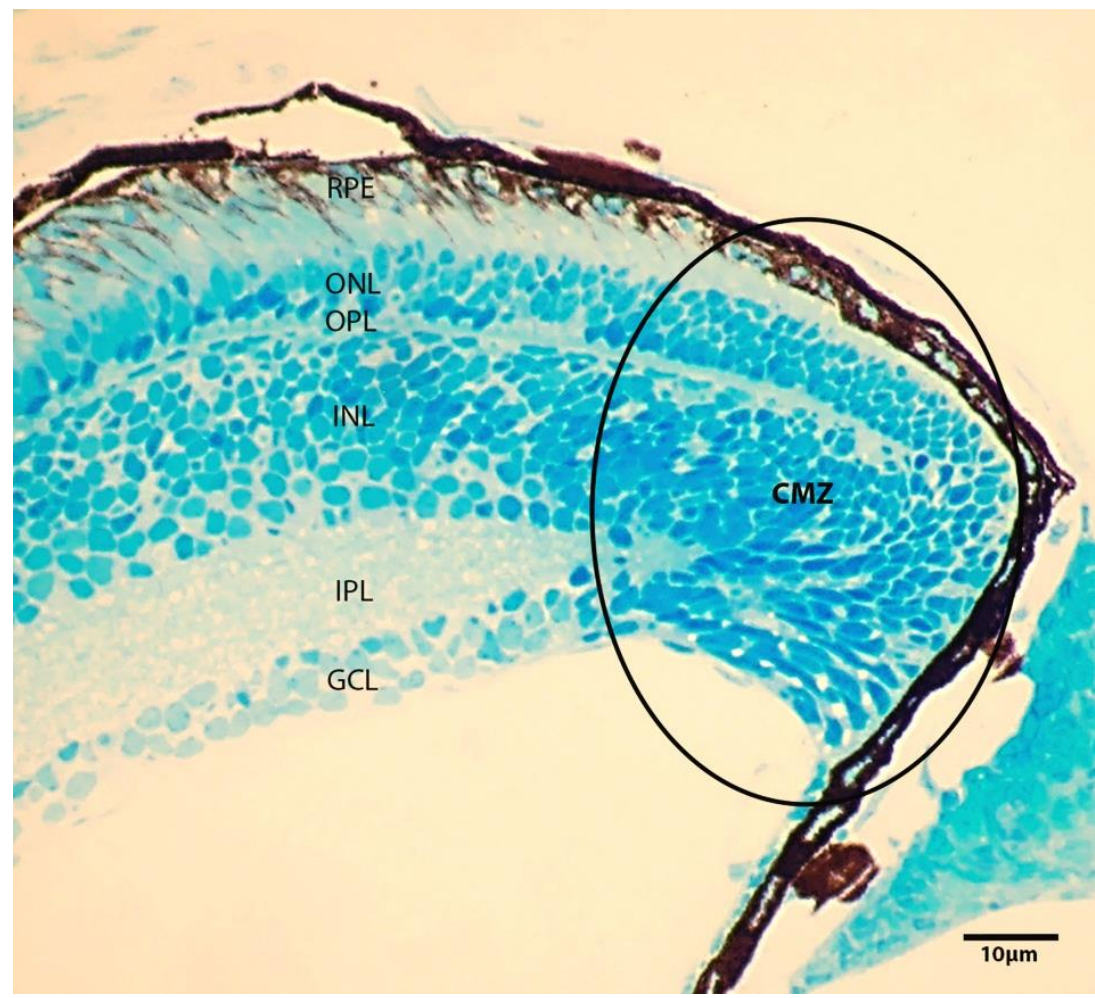

Figure 1. Semi thin section of retina of Austrolebias charrua stained with Methylene blue. CMZ: ciliar marginal zone; RPE: retinal pigmented epithelium; ONL: outer nuclear layer; OPL: outer plexiform layer; INL: inner nuclear layer; IPL: inner plexiform layer; GCL ganglion cell layer.

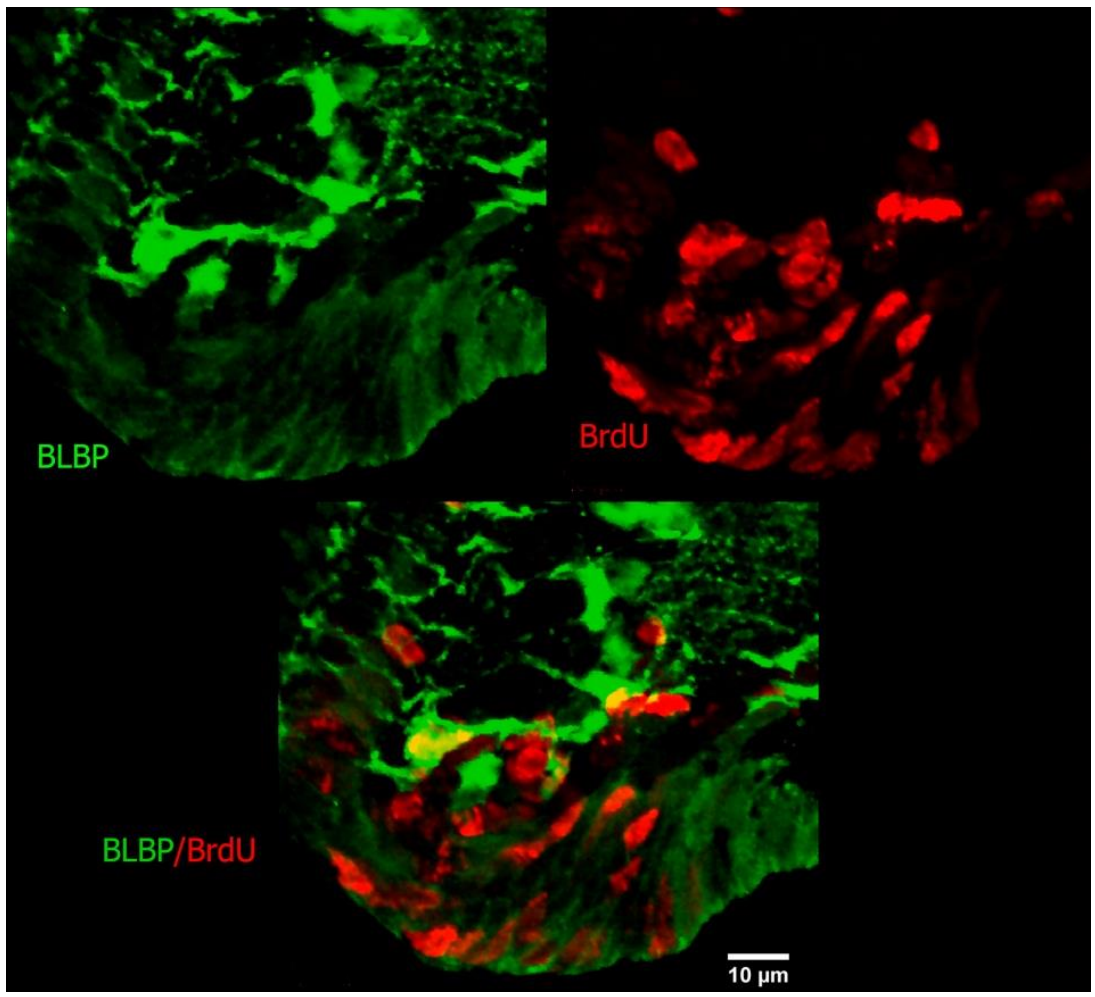

Figure 2. Immunohistochemistry of BrdU (proliferating marker in red), and Brain lipid binding protein (BLBP in green, progenitor marker) in the CMZ. Image from Confocal microscope Olympus FV300. Stack of 20 images taken with 1 micron step. In merged image it is possible to observe the colocalization of BLBP/BrdU in one of the cells in the CMZ. 